\title{
Coding-complete genome sequencing suggests that Newcastle disease virus challenge strain Herts'33 (IVMP) may represent a distinct genotype
}

\author{
Enikő Fehér ${ }^{1}$. Ádám Bálint ${ }^{2} \cdot$ Szilvia Marton ${ }^{1} \cdot$ Krisztina Bali $^{1} \cdot$ Sándor Belák $^{3} \cdot$ Krisztián Bányai $^{1}$ (])
}

Received: 19 June 2019 / Accepted: 15 September 2019 / Published online: 8 November 2019

(c) The Author(s) 2019

\begin{abstract}
We determined the genomic sequence of a Newcastle disease virus (NDV) line obtained directly from the first NDV isolate, named Herts' 33 . This strain shared $\leq 90 \%$ nucleotide sequence identity with the NDV sequences available in the GenBank database, and formed a distinct branch in a phylogenetic tree. This branch may be considered to represent a separate NDV genotype. Our study indicates that investigation of the genomic sequences of old NDV strains that originated from the early outbreaks of Newcastle disease may alter the phylogenetic grouping of the NDV strains and provide data on the evolution of viral genomes over time.
\end{abstract}

Newcastle disease virus (NDV) (species Avian orthoavulavirus 1) belongs to the genus Orthoavulavirus, family Paramyxoviridae, order Mononegavirales. The enveloped virion has a single-stranded, negative-sense RNA genome with a length of $\sim 15.2 \mathrm{~kb}[1,2]$. The viral genome is composed of six genes in the following order: 3'-NP-P-M-F-HN-L-5' (letters designate the nucleoprotein (NP), the phosphoprotein $(\mathrm{P})$, the matrix protein $(\mathrm{M})$, the fusion protein $(\mathrm{F})$, the hemagglutinin-neuraminidase $(\mathrm{HN})$ and the RNA polymerase (L). Based on a number of criteria, the most recent genetic characterization classifies strains belonging to the species Avian orthoavulavirus 1 into genotypes I to XXI and numerous subgenotypes within a subset of genotypes [1].

Handling Editor: Bert K. Rima.

Krisztián Bányai

bkrota@hotmail.com

1 Institute for Veterinary Medical Research, Centre for Agricultural Research, Hungarian Academy of Sciences, Hungária krt. 21, Budapest 1143, Hungary

2 Veterinary Diagnostic Directorate, National Food Chain Safety Office, Budapest, Hungary

3 Department of Biomedical Sciences and Veterinary Public Health (BVF), The OIE Collaborating Centre for the Biotechnology-Based Diagnosis of Infectious Diseases in Veterinary Medicine, Swedish University of Agricultural Sciences (SLU), Uppsala, Sweden
NDV infection may affect a wide range of wild and domestic avian species, causing serious losses during the epizootics [2]. The disease is controlled by vaccination of poultry, typically using live attenuated strains [2, 3]. For example, the viscerotropic Herts' 33 strain was originally isolated from a field sample after an outbreak in 1933 in Hertfordshire (UK) and subsequently became an important strain for control and prevention of Newcastle disease (ND). The mesogenic $\mathrm{H}$ vaccine line was considered a derivate of the Herts'33 field strain and used as a potent live, attenuated vaccine on multiple continents, including Europe [4]. In addition, descendants of the original velogenic Herts'33 strain have been commonly used as challenge strains for testing ND vaccine efficacy.

With the introduction of molecular characterization methods, it has become possible to compare the genetic features of different descendants of Herts'33 and to analyze the homogeneities and differences in their genomes. In a 2003 paper, Czeglédi et al. [4] revealed that the descendant lines of Herts' 33 , including the vaccine strain $\mathrm{H}$, differed in their F protein gene coding sequences, and three genetic groups were identified by phylogenetic analysis. It was hypothesized that the phylogenetic lineage that includes the Weybridge-origin Herts' 33 strain might be a descendant of the isolate from the first ND case, while the Herts' 33 lines that clustered phylogenetically with genotype IV sequences were of unknown origin. Interestingly, the vaccine strain $\mathrm{H}$ clustered with genotype III strains, including other vaccine strains (e.g., Mukteswar) [4]. 
In this study, we determined the complete coding sequence of a lineage Herts'33(W) [4] strain, which has been maintained in eggs and used as a challenge strain at the Institute for Veterinary Medicinal Products, Budapest, Hungary [4]. This strain, referred to here as Herts'33 (IVMP), was purchased in 1999 from the Veterinary Laboratories Agency, Weybridge, and then aliquoted in the Budapest institute. When starting the present study, an aliquot of the original seed was passaged once in eggs to obtain sufficient amount of material for genome sequencing. The viral RNA was extracted using a QIAamp Viral RNA Mini Kit (QIAGEN) and amplified by random RT-PCR as described elsewhere in detail [5]. The amplified cDNA was subjected to sequencing using the Ion Torrent PGM system (Life Technologies). Genome assembly was performed using CLC Genomic Workbench v7.0 (www.qiagenbioinformatics.com/products/ clc-genomics-workbench/). Trimmed sequence reads were mapped onto reference NDV sequences to produce the consensus genome sequence of Herts'33 (IVMP).

The consensus sequence of Herts'33 (IVMP) consisted of 15,166 bases (accession no. MK674396) and lacked sequence information for a 20 nt-long fragment in the 3 , end untranslated region. The genome organization was typical for NDV (Fig. 1), and the cleavage site in the F protein was typical for velogenic and mesogenic NDV strains (aa $\left.{ }^{112} \mathrm{RRQRR} \downarrow \mathrm{F}^{117}\right)[6,7]$.

A BLAST search and phylogenetic analysis $[8,9]$ showed that the closest relative of Herts'33 (IVMP) was the genotype I (class II) strain Ulster/67 (accession no. AY562991), with $90.2 \%$ genome-wide identity. Regarding the complete $\mathrm{F}$ gene, we used the newly recommended method to calculate the average sequence identity among strains; as a result, the $\leq 88.3 \%$ nt sequence identity between the Herts' 33 (IVMP) and the references fell below the cutoff value (i.e., $10 \%$ evolutionary distance) established for genotype discrimination for NDV strains [1, 10]. The topology of a phylogenetic tree based on the F gene also suggested
Fig. 2 Phylogenetic analysis based on full-length fusion gene sequences (A) and representative complete genome sequences (B) using the maximum-likelihood method and the general time-reversible (G) model in MEGA6 software [9], with 100 bootstrap replicates. The Herts'33 (IVMP) sequence is indicated by a black dot

that Herts'33 (IVMP) could be considered a separate genotype (Fig. 2; $[1,10])$. Despite the molecular evidence that Herts'33 (IVMP) represents a unique genetic lineage, unfortunately, independent isolates related to this historic laboratory strain were not available for validation of our genetic classification $[1,10]$.

The strain Herts'33 (IVMP) and a genotype IV strain, Herts/33 (accession no. AY741404), shared 88.1\% genomewide and $85.7 \% \mathrm{~F}$-gene-based nt sequence identity and clustered on different branches in the phylogenetic trees (Figs. 1, $2)$. Additional differences were seen when comparing the lengths of the deduced M and HN proteins (Fig. 1) [6]. The length of the M protein of Herts'33 (IVMP) was identical to that of most of the NDV isolates (364 aa) but shorter than the M protein of Herts/33 (380 aa). The HN protein of Herts'33 (IVMP) was 3 aa longer than the HN protein of other NDV strains (574 aa vs. 571 aa), including Herts/33 (Fig. 1), although additional size variation exists in other strains (e.g., Ulster/67, 616 aa). These data together imply a distinct evolutionary origin of the strains Herts'33 (IVMP) and Herts/33, a finding that corroborates earlier hypotheses [4].

Apparently, some reference NDV strains were maintained at various laboratories for decades without the precise knowledge of their genetic background. In this study, we provide genomic sequence data of an 'old' NDV challenge strain that likely represents an extinct genotype of NDV. This new genome sequence information will be useful to update schemes for classification of NDV isolates and to support more reliable evaluation of experimental data on the genetic diversity of various vaccine and challenge strains of NDV.

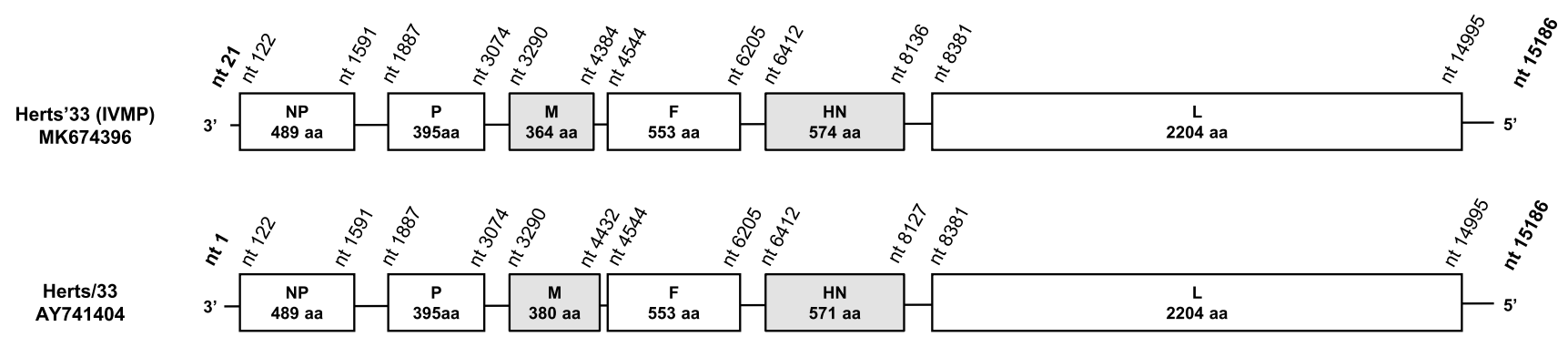

Fig. 1 Schematic representation and comparison of the Herts'33 (IVMP) and Herts/33 genomes. NP, nucleocapsid protein; P, phosphoprotein; M, matrix protein; F, fusion glycoprotein; HN, hemagglu- tinin-neuraminidase protein; L, large or RNA-dependent RNA polymerase protein. The nt positions in Herts'33 (IVMP) were adjusted to those of the fully sequenced strain Herts/33 


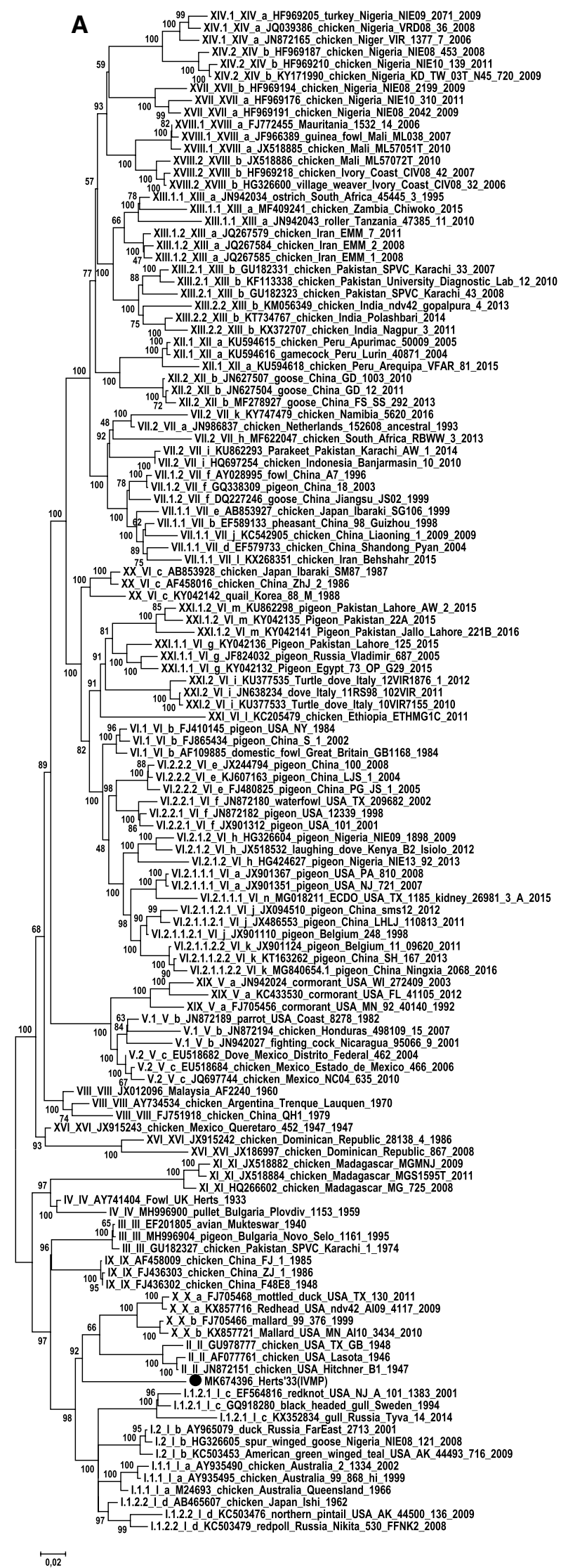

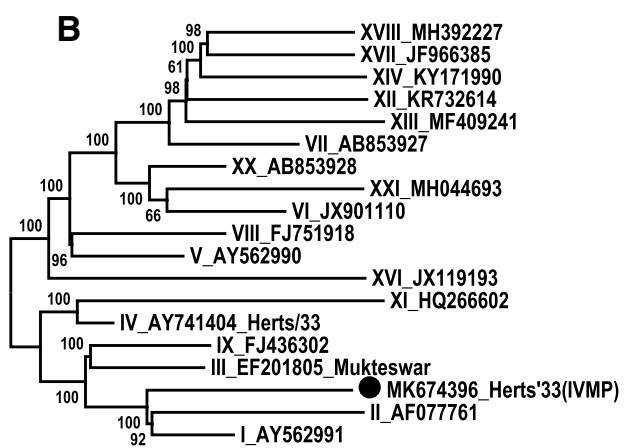

$\stackrel{\longmapsto}{\overrightarrow{0,02}}$ 
Acknowledgements Open access funding provided by MTA Centre for Agricultural Research (MTA ATK).

Author contributions $\mathrm{AB}, \mathrm{SB}$, and $\mathrm{KB}$ conceived the study and provided reagents and materials; $\mathrm{EF}, \mathrm{SM}$, and $\mathrm{KB}$ generated and analyzed data; and EF prepared the first manuscript draft. All authors have read and approved the final version of the manuscript and its submission for publication.

Funding Support was obtained from the Bolyai János Fellowship program awarded by the Hungarian Academy of Sciences (EF, SM), from the Swedish Research Council FORMAS Strong Research Environments project, nr 2011-1692, "BioBridges" (SB), and from the Hungarian Scientific Research Fund (NKFI-OTKA, K120201) (KB).

\section{Compliance with ethical standards}

Conflict of interest The authors declare no conflict of interest.

Open Access This article is distributed under the terms of the Creative Commons Attribution 4.0 International License (http://creativeco mmons.org/licenses/by/4.0/), which permits unrestricted use, distribution, and reproduction in any medium, provided you give appropriate credit to the original author(s) and the source, provide a link to the Creative Commons license, and indicate if changes were made.

\section{References}

1. Dimitrov KM, Abolnik C, Afonso CL, Albina E, Bahl J, Berg M, Briand FX, Brown IH, Choi KS, Chvala I, Diel DG, Durr PA, Ferreira HL, Fusaro A, Gil P, Goujgoulova GV, Grund C, Hicks JT, Joannis TM, Torchetti MK, Kolosov S, Lambrecht B, Lewis NS, Liu H, Liu H, McCullough S, Miller PJ, Monne I, Muller CP, Munir M, Reischak D, Sabra M, Samal SK, de Almeida SR, Shittu I, Snoeck CJ, Suarez DL, Van Borm S, Wang Z, Wong FYK (2019) Updated unified phylogenetic classification system and revised nomenclature for Newcastle disease virus. Infect Genet Evol. 74:103917. https://doi.org/10.1016/j.meegid.2019.103917

2. Bello MB, Yusoff K, Ideris A, Hair-Bejo M, Peeters BPH, Omar AR (2018) Diagnostic and vaccination approaches for Newcastle Disease Virus in poultry: the current and emerging perspectives. Biomed Res Int 2018:7278459

3. Mayers J, Mansfield KL, Brown IH (2017) The role of vaccination in risk mitigation and control of Newcastle disease in poultry. Vaccine 35:5974-5980

4. Czeglédi A, Wehmann E, Lomniczi B (2003) On the origins and relationships of Newcastle disease virus vaccine strains Hertfordshire and Mukteswar, and virulent strain Herts'33. Avian Pathol 32:271-276

5. Bányai K, Kemenesi G, Budinski I, Földes F, Zana B, Marton S, Varga-Kugler R, Oldal M, Kurucz K, Jakab F (2017) Candidate new rotavirus species in Schreiber's bats, Serbia. Infect Genet Evol 48:19-26

6. de Leeuw OS, Koch G, Hartog L, Ravenshorst N, Peeters BP (2005) Virulence of Newcastle disease virus is determined by the cleavage site of the fusion protein and by both the stem region and globular head of the haemagglutinin-neuraminidase protein. J Gen Virol 86:1759-1769

7. Gould AR, Hansson E, Selleck K, Kattenbelt JA, Mackenzie M, Della-Porta AJ (2003) Newcastle disease virus fusion and haemagglutinin-neuraminidase gene motifs as markers for viral lineage. Avian Pathol 32:361-373

8. Altschul SF, Gish W, Miller W, Myers EW, Lipman DJ (1990) Basic local alignment search tool. J Mol Biol 215:403-410

9. Tamura K, Stecher G, Peterson D, Filipski A, Kumar S (2013) MEGA6: molecular evolutionary genetics analysis version 6.0. Mol Biol Evol 30:2725-2729

10. Diel DG, da Silva LH, Liu H, Wang Z, Miller PJ, Afonso CL (2012) Genetic diversity of avian paramyxovirus type 1: proposal for a unified nomenclature and classification system of Newcastle disease virus genotypes. Infect Genet Evol 12:1770-1779

Publisher's Note Springer Nature remains neutral with regard to jurisdictional claims in published maps and institutional affiliations. 